\title{
Steady-state and transient behavior in dynamic atomic force microscopy
}

\author{
Tino Wagner* ${ }^{*}$ \\ Nanotechnology Group, ETH Zürich, Säumerstrasse 4, 8803 Rüschlikon, Switzerland
}

(Dated: January 29, 2019)

\begin{abstract}
We discuss the influence of external forces on the motion of the tip in dynamic atomic force microscopy (AFM). First, a compact solution for the steady-state problem is derived employing a Fourier approach. Founding on this solution, we present an analytical framework to describe the transient behavior of the tip after perturbations of tip-sample forces and the excitation signal. The static and transient solutions are then combined to obtain the baseband response of the tip, i.e., the deflection signal demodulated with respect to the excitation. The baseband response generalizes the amplitude and phase response of the tip, and we use it to find explicit formulas describing the amplitude and phase modulation following the influence of external forces on the tip. Finally, we apply our results to obtain an accurate dynamic model of the amplitude controller and phase-locked loop (PLL) driving the cantilever in a frequency modulated AFM setup. A special emphasis is put on discussing the tip response in environments of high damping, such as ambient or liquid.
\end{abstract}

\section{INTRODUCTION}

Atomic force microscopy is an extremely versatile microscopy technique in surface science, which utilizes the force between a tip moving above the surface to obtain a highly resolved topographic image. Since its invention in 1986 [1, there have been numerous improvements to the technique. First and foremost, there has been a transition to dynamic operation modes 2, in which the tip, mounted on a force sensor such as a cantilever, is forced to oscillate near or at the resonance frequency. The tip-sample force changes the resonant behavior of the force sensor, allowing one to detect changes of the tip-sample interaction from changes of the detected oscillation amplitude 3] (amplitude modulated, AM-AFM) or resonance frequency [4] (FM-AFM) of the cantilever.

In addition to topography measurements, complementary information can be obtained from the tip-surface interaction. For example, the power dissipated by the tip can be measured and related to viscoelastic damping and frictional forces [5, 6. The tip-sample force also contains magnetic [7, electrostatic [8, and near-field optical [9] information about the sample. Individual force contributions can be modulated by an external stimulus, such as an external voltage bias or pulsed-light illumination, to facilitate separation from other contributions in the frequency domain, and to enhance their detection sensitivity. The electrostatic force can be modulated and nullified to obtain surface potential maps (Kelvin probe force microscopy, KFM) [10, 11.

The theory of dynamic AFM describes the effects of external forces on the motion of the AFM tip mounted on a cantilever. While the theory of specific detection schemes, such as AM-AFM [3, 12] and FM-AFM [4, 13, 14, is well established, a general theory of steady-state operation with a minimum number of assumptions was only developed recently 15$]$.

\footnotetext{
* E-mail: tiwagner@ethz.ch
}

In this paper, we describe a unified theory of dynamic AFM that extends beyond the steady-state solution by also considering the transient response to perturbations.

First, we focus on the steady-state solution for excitation frequencies far below and at resonance of the cantilever. We approach this problem from the frequency domain, allowing us to make the simple connection from Fourier components of the tip-sample force to the cantilever response at different harmonics.

For excitation close to resonance, we present analytical expressions under the so-called harmonic approximation [15], which assumes a purely sinusoidal cantilever movement and thereby disregards harmonics caused by the non-linear tip-sample force. As discussed later, this approximation is justified for most force sensors and operating conditions. We demonstrate the generality of the obtained steady-state solution to explain the operational bistabilty in AM-AFM 16 18.

Next, we go beyond the steady state and investigate the transient cantilever response after perturbations of the tip-sample force or the sinusoidal excitation. The behavior of transients is derived analytically in the Laplace domain, which facilitates straightforward interpretation in the frequency domain and enables the calculation of the response to an arbitrarily-shaped perturbation. We show how perturbations of the external force at different frequencies affect the tip movement, and how frequency mixing due to the non-linear tip-sample interaction can be exploited.

The transients are then used to derive the baseband dynamics of the tip, which are experimentally accessible by coherent demodulation of the cantilever deflection at the excitation frequency. The complex baseband signal contains information about the amplitude and phase modulation caused by the tip-sample force. Through the baseband signal, we derive transfer functions from frequency components of the external force to the amplitude, phase, and frequency response of the cantilever.

In practice, these transfer functions are important for correct tuning of feedback loops and signal-to-noise analysis of advanced AFM techniques, such as multi- 
frequency [19], heterodyne [20] and multi-harmonic [21] modes of operation.

We use the derived transfer functions to determine the actual closed-loop behavior of the PLL and amplitude controller in FM-AFM. The behavior of the PLL is discussed for different controller gains and quality factors of the cantilever.

Lastly, we highlight how the theory presented in this paper benefits AFM simulators. The separation of the calculation into steady-state and transients permits a large speedup compared to computationally expensive time-domain simulations of the full equation of motion. The simulation can be done directly at the baseband level, which mitigates the calculation of high-frequency perturbations that are filtered out for common operational modes.

\section{EQUATION OF MOTION}

The motion of the tip of an atomic force microscope is well approximated by the behavior of a damped harmonic oscillator 22. In the vicinity of the surface, the tip-sample force $F_{\mathrm{ts}}$ acts on the tip at the position $z_{\mathrm{t}}$

$$
\ddot{z}_{\mathrm{t}}+\frac{\omega_{0}}{Q} \dot{z}_{\mathrm{t}}+\omega_{0}^{2}\left(z_{\mathrm{t}}-z_{\mathrm{b}}\right)=\frac{\omega_{0}^{2}}{k} F_{\mathrm{ts}}\left(z_{\mathrm{ts}}, \dot{z}_{\mathrm{ts}}, t\right),
$$

where the eigenfrequency $\omega_{0}$ and intrinsic dissipation, expressed as the quality factor $Q$, determine the shape of the resonance, and $k$ is the spring constant of the cantilever. $z_{\mathrm{b}}$ is base position of the cantilever and $z_{\mathrm{ts}}$ is the tip-sample distance.

Experimentally, the deflection $q=z_{\mathrm{t}}-z_{\mathrm{b}}$ of the tip rather than its position $z_{\mathrm{t}}$ is measured. The corresponding equation of motion is therefore

$$
\ddot{q}+\frac{\omega_{0}}{Q} \dot{q}+\omega_{0}^{2} q=\omega_{0}^{2} a(t)+\frac{\omega_{0}^{2}}{k} F_{\mathrm{ts}}\left(z_{\mathrm{ts}}, \dot{z}_{\mathrm{ts}}, t\right),
$$

where $a(t)$ is the excitation of the tip resulting from the movement of the base 22]:

$$
a(t)=-\frac{\ddot{z}_{\mathrm{b}}}{\omega_{0}^{2}}-\frac{\dot{z}_{\mathrm{b}}}{Q \omega_{0}} .
$$

For a sinusoidal displacement of the base at the frequency $\omega$, eq. (3) corresponds to a static change in amplitude and phase by $\left(\omega / \omega_{0}\right)^{2}$ and $\arctan \left(-\omega_{0} / \omega Q\right)$, respectively. In the limit of low intrinsic damping and drive near resonance $\left(\omega \approx \omega_{0}, Q \rightarrow \infty\right)$, the amplitude and phase are left unchanged, and there is no difference exciting the base or tip [22, 23. For the sake of clarity, we consider in the following a direct excitation of the tip via $a(t)$ rather than a movement of the base. This has no implication on the generality of the results; however, the effects of eq. (3) must be considered separately when operating off resonance or in an environment of high damping.

\section{STEADY-STATE SOLUTION}

In the steady-state, transients due to the explicit time dependency of $F_{\mathrm{ts}}$ must have decayed. $F_{\mathrm{ts}}\left(z_{\mathrm{ts}}(t), \dot{z}_{\mathrm{ts}}(t)\right)$ is, in general, a non-linear function of the tip trajectory. Hence, for sinusoidal excitation of the oscillator at the frequency $\omega$ with an amplitude $a_{0}$,

$$
a(t)=a_{0} \cos (\omega t),
$$

a response of the deflection $q(t)$ at multiple harmonics $(\omega, 2 \omega, 3 \omega, \ldots)$ is expected.

Because the excitation signal $a(t)=a(t+T)$ is periodic in time with the period $T=2 \pi / \omega$, the tip trajectory $z_{\mathrm{ts}}$ and the tip-sample force $F_{\mathrm{ts}}$ must also be periodic functions with the same period $T$. Therefore, $q$ and $F_{\text {ts }}$ can be written as a Fourier series,

$$
q(t)=\sum_{n=-\infty}^{\infty} \hat{q}_{n} e^{i n \omega t} \text { and } F_{\mathrm{ts}}(t)=\sum_{n=-\infty}^{\infty} \hat{f}_{n} e^{i n \omega t}
$$

Inserting eq. (4) and eq. (5) in eq. (2), we obtain a system of equations for the Fourier coefficients of the deflection $\hat{q}_{n}$,

$$
\begin{aligned}
k \hat{q}_{0} & =\hat{f}_{0}, \\
k \hat{q}_{ \pm 1}\left[-\omega^{2} \pm i \frac{\omega \omega_{0}}{Q}+\omega_{0}^{2}\right] & =k \omega_{0}^{2} \frac{a_{0}}{2}+\omega_{0}^{2} \hat{f}_{ \pm 1}, \\
k \hat{q}_{ \pm n}\left[-( \pm n \omega)^{2} \pm i \frac{n \omega \omega_{0}}{Q}+\omega_{0}^{2}\right] & =\omega_{0}^{2} \hat{f}_{ \pm n} .
\end{aligned}
$$

Equation (6) describes the static deflection of the cantilever, which is related to $\hat{f}_{0}$ by the spring constant $k$. Equation (7) describes the response of the fundamental harmonic of the deflection, $\hat{q}_{ \pm 1}$. This response is driven both by the excitation force $k a_{0}$ at the drive frequency $\omega$, and the Fourier component $\hat{f}_{ \pm 1}$. The Fourier coefficients for higher harmonics $|n|>1$ follow from eq. (8).

For the solution of eqs. (6) to (8), the tip trajectory obtained from the Fourier components $\hat{q}_{n}$ of the deflection, is required to calculate the Fourier coefficients $\hat{f}_{n}$ of $F_{\mathrm{ts}}\left(z_{\mathrm{ts}}(t), \dot{z}_{\mathrm{ts}}(t)\right)$; likewise, $\hat{q}_{n}$ requires all $\hat{f}_{n}$ to be known. Therefore, in general, a self-consistent approach must be employed to solve for $\hat{q}_{n}$. An efficient numerical solution is possible in many circumstances by Newton's method. Since $F_{\mathrm{ts}}$ is a non-linear function of $z_{\mathrm{ts}}$, there can be multiple solutions, and the resulting deflection $q$ may sensibly depend on the initial conditions. For the numerical solution, special care must be taken when the tip movement becomes unstable upon approach and retract. Experimentally, this is the case when 'snap-into-contact' and 'pull-off' events are observed [22], i.e., when the force gradient $k_{\mathrm{ts}}$ acting on the tip exceeds the spring constant $k$ of the cantilever. In such cases, the solution of eqs. (6) to (8) in the frequency domain can be cumbersome, whereas a time-domain solution of the equation of motion, eq. (2), is usually straightforward. However, as 
shown below, an analytical solution is possible when the fundamental harmonic dominates the cantilever movement.

The number of significant harmonics observed in the cantilever deflection depends on the relative drive frequency $\omega / \omega_{0}$. For an oscillator driven near resonance, i.e., $\omega / \omega_{0} \approx 1$, harmonics of the drive frequency are rarely observed. This can be seen from eqs. (7) and (8): to obtain each $\hat{q}_{n}, \hat{f}_{n}$ is divided by a gain factor on the left hand side. For $\omega \approx \omega_{0}$, the gain ratio of the fundamental to the $n$-th harmonic is $\sqrt{Q^{2}\left(1-n^{2}\right)^{2}+n^{2}}$. Hence, the harmonics at $n \omega_{0}$ are off-resonance and diminish with increasing order $n$ and quality factor $Q$. For reasonable quality factors $Q$ exceeding the order $n$, i.e. $Q \gg n$, harmonics decrease in amplitude as $1 / Q\left(n^{2}-1\right)$. Even in the limit of a highly overdamped oscillator, $Q \rightarrow 0$, harmonics still decrease with increasing order as $1 / n$. For harmonics to be relevant and within the detection limits, the oscillation amplitude must be sufficiently high to generate strong harmonics, and the quality factor must be very low [24, 25].

When the oscillator is instead driven off-resonance at frequencies $\omega$ well below the eigenfrequency $\omega_{0}$, the $n$ th harmonic is attenuated for $n \omega \gg \omega_{0}$ and amplified near resonance. This is the case for AFM techniques based on fast acquisition of force-distance curves [26, 27]. In this case, the reconstruction of the tip-sample force during each oscillation cycle is possible via eqs. (6) to (8) from the deflection $\hat{q}_{n}$ and the parameters of the freely oscillating cantilever. In doing so, care must be taken to avoid amplifying noise for higher harmonics beyond $\omega_{0}$.

Alternatively, the non-linear tip-sample force can be reconstructed from its harmonics by driving the cantilever at more than one frequency near the resonance. Because of frequency mixing, intermodulation products appear in the deflection signal [22, 28]. By appropriate choice of the driving frequencies and their spacing, many intermodulation products are within the resonance, such that they can be detected with a high signal-to-noise ratio.

In the following, we consider the case where the fundamental harmonic dominates the deflection signal. As reasoned above, this harmonic approximation is valid in most experiments with a single-tone excitation near the cantilever resonance. Experimentally, the validity of this approximation can be verfied by observing the power spectrum of $q(t)$ for harmonics of $\omega$.

Under the harmonic approximation, the deflection and tip-sample distance are

$$
\begin{aligned}
q & =q_{\mathrm{s}}+A \cos (\omega t+\varphi) \\
z_{\mathrm{ts}} & =z_{\mathrm{b}}+q=z_{\mathrm{c}}+A \cos (\omega t+\varphi),
\end{aligned}
$$

where $q_{\mathrm{s}}$ is the static deflection, $z_{\mathrm{c}}=z_{\mathrm{b}}+q_{\mathrm{s}}$ is the average (center) tip distance, and $A$ and $\varphi$ are the oscillation amplitude and phase, respectively.
The static deflection is governed by the time average of the tip-sample force,

$$
\begin{aligned}
\hat{f}_{0} & =\frac{1}{T} \int_{-T / 2}^{T / 2} \mathrm{~d} t F_{\mathrm{ts}}\left(z_{\mathrm{ts}}(t), \dot{z}_{\mathrm{ts}}(t)\right) \\
& =\frac{1}{\pi} \int_{-A}^{A} \mathrm{~d} q \frac{F_{\mathrm{ts}}\left(z_{\mathrm{c}}+q\right)}{\sqrt{A^{2}-q^{2}}}=:\left\langle F_{\mathrm{ts}}\right\rangle .
\end{aligned}
$$

The steady-state solution, eq. (9), requires

$$
\hat{q}_{0}=q_{\mathrm{s}} \quad \text { and } \quad \hat{q}_{ \pm 1}=A \exp ( \pm i \varphi) / 2
$$

For nonzero amplitudes $A$, we can always write $\hat{f}_{ \pm 1}$ as a product of a complex number $\hat{k}_{ \pm 1}$ and $\hat{q}_{ \pm 1}$, corresponding to scaling and rotation of the complex force gradient $\hat{k}_{ \pm 1}$ :

$$
\hat{f}_{ \pm 1}=\hat{k}_{ \pm 1} \hat{q}_{ \pm 1}
$$

Because $q(t)$ and $F_{\mathrm{ts}}(t)$ are real signals, we can restrict the further analysis to non-negative Fourier coefficients. From the left side of eq. (7), we see that the real and imaginary components of $k_{1}$ change the eigenfrequency and damping to effective new values:

$$
\begin{gathered}
\omega_{0}^{2} \rightarrow \omega_{0}^{2}\left(1-\operatorname{Re} \hat{k}_{1} / k\right) \\
\frac{1}{Q} \rightarrow \frac{1}{Q}-\frac{\omega_{0}}{\omega} \operatorname{Im} \hat{k}_{1} / k
\end{gathered}
$$

$\hat{k}_{1}$ is related to the tip-sample force as

$$
\hat{k}_{1}=\frac{2 \hat{f}_{1}}{A e^{i \varphi}}=\frac{2}{A T} \int_{t_{0}}^{t_{0}+T} \mathrm{~d} t F_{\mathrm{ts}}(t) e^{-i(\omega t+\varphi)} .
$$

For the real and imaginary parts of $\hat{k}_{1}$, we find

$$
\begin{aligned}
& \operatorname{Re} \hat{k}_{1}=\frac{2}{A T} \int_{t_{0}}^{t_{0}+T} \mathrm{~d} t F_{\mathrm{ts}}(t) \cos (\omega t+\varphi) \\
& \operatorname{Im} \hat{k}_{1}=-\frac{2}{A T} \int_{t_{0}}^{t_{0}+T} \mathrm{~d} t F_{\mathrm{ts}}(t) \sin (\omega t+\varphi)
\end{aligned}
$$

Equations (17) and (18) suggest that changes of eigenfrequency and damping are determined by the in-phase and quadrature components of $F_{\text {ts }}$ with respect to the tip oscillation. The integrals in eqs. (17) and (18) are zero if $F_{\mathrm{ts}}(t-\varphi / \omega)$ is odd or even in $t$, respectively. As suggested by Sader et al. [14, this motivates splitting $F_{\text {ts }}$ into even and odd parts, which govern changes of the eigenfrequency and damping, respectively.

The odd force can be written in terms of a product of an even and odd function. Naturally, choosing the tip velocity as the odd function, this results in the generalized damping coefficient [14, 29] $\gamma_{\mathrm{ts}}$,

$$
F_{\text {odd }}\left(z_{\mathrm{ts}}, \dot{z}_{\mathrm{ts}}\right)=-\gamma_{\mathrm{ts}}\left(z_{\mathrm{ts}}\right) \dot{z}_{\mathrm{ts}}
$$


Using the even and odd components $F_{\text {even }}$ and $F_{\text {odd }}$ of $F_{\mathrm{ts}}$, and substituting the tip trajectory $z_{\mathrm{ts}}(t)$, we obtain

$$
\begin{aligned}
\operatorname{Re} \hat{k}_{1} & =\frac{2}{\pi A^{2}} \int_{-A}^{A} \mathrm{~d} q \frac{F_{\text {even }}\left(z_{\mathrm{c}}+q\right) q}{\sqrt{A^{2}-q^{2}}} \\
& =\frac{2}{\pi A^{2}} \int_{-A}^{A} \mathrm{~d} q k_{\mathrm{ts}}\left(z_{\mathrm{c}}+q\right) \sqrt{A^{2}-q^{2}} \\
& =:\left\langle k_{\mathrm{ts}}\right\rangle \text { and } \\
\operatorname{Im} \hat{k}_{1} & =-\omega \frac{2}{\pi A^{2}} \int_{-A}^{A} \mathrm{~d} q \gamma_{\mathrm{ts}}\left(z_{\mathrm{c}}+q\right) \sqrt{A^{2}-q^{2}} \\
& =:-\omega\left\langle\gamma_{\mathrm{ts}}\right\rangle,
\end{aligned}
$$

with the force gradient $k_{\mathrm{ts}}=\partial F_{\text {even }} / \partial z$, and $\left\langle k_{\mathrm{ts}}\right\rangle$ and $\left\langle\gamma_{\mathrm{ts}}\right\rangle$ are the effective force gradient and damping coefficient, respectively. Unlike $\left\langle F_{\mathrm{ts}}\right\rangle$, which represents a simple time average of the force and whose main contributions are due to the turning points of the oscillation, the time averages for $\left\langle k_{\mathrm{ts}}\right\rangle$ and $\left\langle\gamma_{\mathrm{ts}}\right\rangle$ are weighted by the oscillation itself, which results in a weighting over a semicircle around $z_{\mathrm{c}}$.

The formula for $\left\langle k_{\mathrm{ts}}\right\rangle$ was first derived by Giessibl [13] using a Hamilton-Jacobi perturbation approach and later rewritten as a weighted average over $k_{\mathrm{ts}}$ by integration by parts [30. A similar Fourier ansatz to the one shown here has been employed before for FM-AFM [14, 29, 31] and AM-AFM [32].

Recently, from a consideration of the average kinetic energy and average power of the resonator, Söngen et al. [15] derived three equations relating the excitation parameters $\left(a_{0}, \omega\right)$ and observables $\left(q_{\mathrm{s}}, A, \varphi\right)$ of typical dynamic AFM experiments to the averages $\left\langle F_{\mathrm{ts}}\right\rangle,\left\langle k_{\mathrm{ts}}\right\rangle$, and $\left\langle\gamma_{\mathrm{ts}}\right\rangle$. These equations also follow naturally from eqs. (6) and (7). Inserting the observables from eq. (12) and using the averages defined in eqs. (11), (20) and (21), we obtain:

$$
\begin{aligned}
\left\langle F_{\mathrm{ts}}\right\rangle / k & =q_{\mathrm{s}} \\
\left\langle k_{\mathrm{ts}}\right\rangle / k & =1-\left(\omega / \omega_{0}\right)^{2}-\frac{a_{0}}{A} \cos \varphi \\
& \approx-2 \frac{\Delta \omega}{\omega_{0}}-\frac{a_{0}}{A} \cos \varphi \\
\left\langle\gamma_{\mathrm{ts}}\right\rangle / k & =-\frac{1}{\omega_{0} Q}-\frac{a_{0}}{\omega A} \sin \varphi .
\end{aligned}
$$

In eq. 22c we have used $\omega=\omega_{0}+\Delta \omega$ and approximated for small frequency shifts $\Delta \omega$. The equations are useful to quantitatively compare measurements obtained by different AFM implementations, as demonstrated for AM- and FM-AFM spectroscopy data 15. It should be noted that the averages themselves are non-linear functions of distance $z_{\mathrm{c}}$ and amplitude $A$; a direct comparison is therefore only possible for data collected with a similar amplitude, or after deconvolving the averages to reconstruct the tip-sample force [14, 15, 33.

In similar form, eqs. (22b) and (22d) have been derived before by San Paulo and Garcia [12] to obtain a general theory of AM-AFM. The relation of dissipation and oscillation phase, eq. $22 \mathrm{~d}$, was first given by Cleveland et al. [5].

Eliminating the phase in eqs. 22b and $22 \mathrm{~d}$, we obtain an algebraic equation for the oscillation amplitude $A$ as a function of excitation amplitude $a_{0}$ and frequency $\omega:$

$$
\frac{a_{0}}{A}=\sqrt{\left[1-\left(\frac{\omega}{\omega_{0}}\right)^{2}-\frac{\left\langle k_{\mathrm{ts}}\right\rangle}{k}\right]^{2}+\left[\frac{\omega}{\omega_{0} Q}+\frac{\omega\left\langle\gamma_{\mathrm{ts}}\right\rangle}{k}\right]^{2}}
$$

For fixed excitation parameters $a_{0}$ and $\omega$, the solution of eq. 23) are the oscillation amplitudes $A$ allowed at the distance $z_{\mathrm{c}}$.

The time-averaged power dissipated by the tip, $\left\langle P_{\text {tip }}\right\rangle$, is related to the driving frequency $\omega$, steady-state amplitude $A$, and the tip-sample damping coefficient $\left\langle\gamma_{\mathrm{ts}}\right\rangle$ :

$$
\left\langle P_{\mathrm{tip}}\right\rangle=-\left\langle F_{\text {odd }} \dot{z}_{\mathrm{ts}}\right\rangle=\left\langle\gamma_{\mathrm{ts}} \dot{z}_{\mathrm{ts}}^{2}\right\rangle=\omega^{2} A^{2}\left\langle\gamma_{\mathrm{ts}}\right\rangle / 2 .
$$

Motivated by the substitution in eqs. (14) and (15), the original non-linear tip-sample force $F_{\text {ts }}$ can be approximated in terms of the averages $\left\langle F_{\mathrm{ts}}\right\rangle,\left\langle k_{\mathrm{ts}}\right\rangle$, and $\left\langle\gamma_{\mathrm{ts}}\right\rangle$. Therefore, $F_{\mathrm{ts}}$ in eq. (2) may be substituted with

$$
F_{\mathrm{ts}} \approx\left\langle F_{\mathrm{ts}}\right\rangle(t)+\left\langle k_{\mathrm{ts}}\right\rangle(t)\left(q-q_{\mathrm{s}}\right)-\left\langle\gamma_{\mathrm{ts}}\right\rangle(t) \dot{q}
$$

Note that the individual terms may still be treated as weak functions of time, since the averages in eqs. (11), (20) and 21 can be taken as short as a single period of oscillation. Equation 25 may be considered an equivalent linearization 34 of the non-linear equation of motion. The solution derived from the harmonic approximation is therefore equivalent to the one obtained using the Krylov-Bogoliubov averaging method [34, 35. Equation 25 approximates the tip-sample force by its Fourier coefficients $\hat{f}_{0}$ and $\hat{f}_{ \pm 1}$ only.

As an application of the steady-state solution discussed above, we show in fig. 1 the averages $\left\langle F_{\mathrm{ts}}\right\rangle$ and $\left\langle k_{\mathrm{ts}}\right\rangle$ as function of amplitude $A$ and distance of closest approach, $z_{\mathrm{c}}-A$, together with the phase and excitation calculated from eqs. (22b) and $22 \mathrm{~d}$ ). Tip-sample interactions are given by a Derjaguin-Muller-Toporov (DMT) contact model combined with van der Waals (vdW) interactions, which is commonly employed in literature [2, 12, 17, 22]:

$$
F_{\mathrm{ts}}(z)= \begin{cases}-\frac{H R}{6 z^{2}} & z>c \\ -\frac{H R}{6 c^{2}}+\frac{4}{3} E^{*} \sqrt{R(c-z)^{3}} & z \leq c\end{cases}
$$

Here, $H$ is the Hamaker constant, $E^{*}$ is the effective Young's modulus of the tip-sample system, and $c$ is the distance at which the tip leaves the contact regime.

Notably, fig. 1(c) shows the bistability commonly observed under AM-AFM operation [12, 17, 18]: for a cantilever which is driven at fixed excitation $a_{0}$, there can be several possible distances $z_{\mathrm{c}}$ resulting in an oscillation with the amplitude $A$. 
a

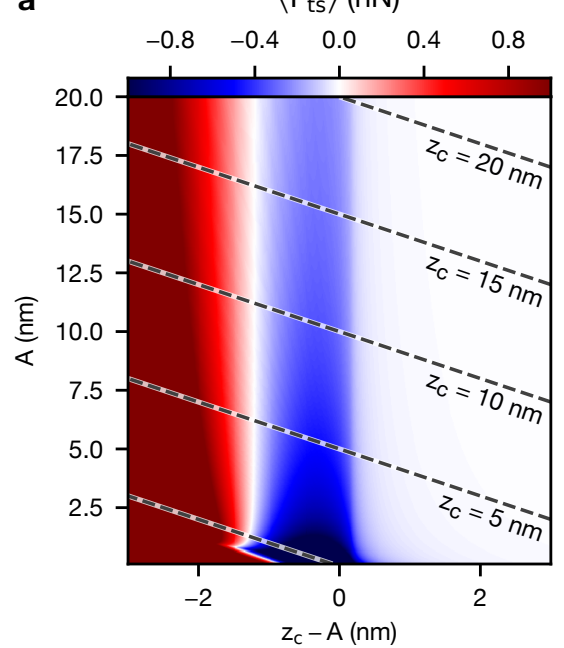

b

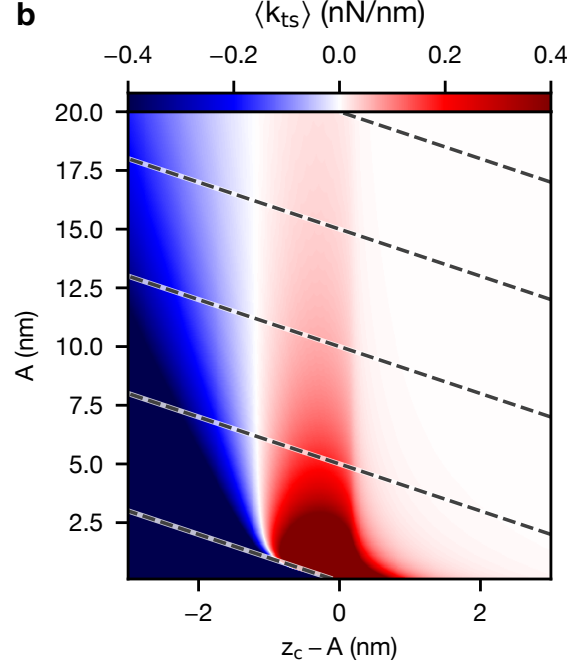

c

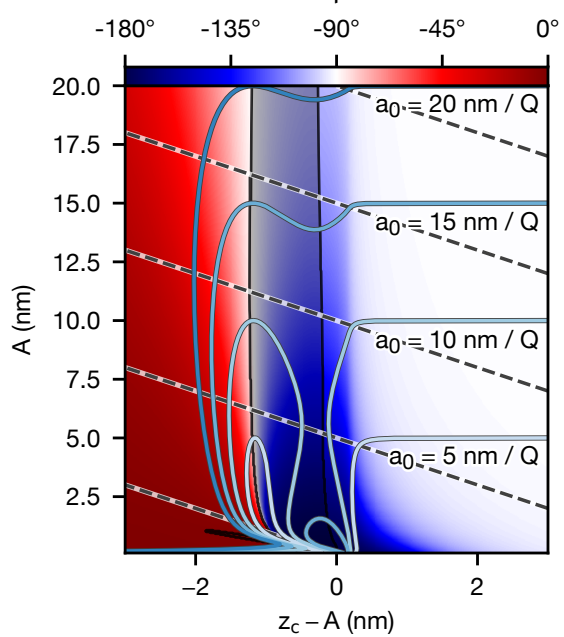

FIG. 1. Effective (a) tip-sample force and (b) force gradient as a function of amplitude $A$ and distance of closest approach $z_{\mathrm{c}}-A$. Tip-sample interactions are given by a vdW-DMT model as parameterized in San Paulo and Garcia $[12]\left(H=6.4 \times 10^{-20} \mathrm{~J}\right.$, $E^{*}=1 \mathrm{GPa}, c=0.17 \mathrm{~nm}, R=10 \mathrm{~nm}$ ). Dashed lines indicate contours of constant average tip-sample distance $z_{\mathrm{c}}$. (c) Phase and contours of constant excitation resulting from eqs. 22b and 23 for a cantilever with $\omega_{0}=\omega=2 \pi 325 \mathrm{kHz}, Q=400$, and $k=40 \mathrm{~N} \mathrm{~m}^{-1}$. Note that for constant excitation $a_{0}$, a steady-state oscillation with a certain amplitude $A$ is possible at multiple distances. The region shaded in gray indicates solutions which are unstable, that is, small perturbations result in a jump to a nearby stable solution.

Not every combination of $A$ and $z_{\mathrm{c}}$ is stable, however. For the gray shaded area in fig. 1(c), a small perturbation is sufficient to drive the oscillation to the nearest stable branch. This region was found by a numerical simulation of the equation of motion, eq. 2, together with the effective $F_{\mathrm{ts}}$, eq. 25). The averages are calculated efficiently using Chebyshev-Gauss quadrature and are precomputed on a grid as shown in fig. 1(a) and (b). The equation of motion is evaluated at the baseband (c.f. section V), such that the resulting amplitude and phase are available without further signal processing.

For large free amplitudes (> $10 \mathrm{~nm})$, net-repulsive tip-sample interactions are favored, because there exists no net-attractive branch at typical imaging setpoints of $80-90 \%$. For smaller amplitudes $(<10 \mathrm{~nm})$, there are both stable net-attractive and stable net-repulsive branches for most amplitudes below the free amplitude. Operation in the net-attractive regime is favored increasingly, because the separation of the stable branches is increased, and strong perturbations are required to facilitate a jump between them.

\section{TRANSIENTS}

Next, we discuss the response of the tip deflection $q(t)$ to changes of the external forces. To this end, it is instructive to work with the Laplace transform $\tilde{q}(s)=\mathcal{L}\{q\}(s)$ instead, because the time-domain behavior is then reflected in algebraic expressions of the complex variable $s=\sigma+i \omega$.
Far from the surface, the cantilever behavior is found from the Laplace transform of eq. (2) with $F_{\mathrm{ts}} \equiv 0$,

$$
\begin{aligned}
\tilde{q}(s) & =G_{0}(s) \tilde{a}(s) \text { with } \\
G_{0}(s) & =\frac{\omega_{0}^{2}}{s^{2}+\omega_{0} s / Q+\omega_{0}^{2}},
\end{aligned}
$$

where we denote $\tilde{q}(s)$ and $\tilde{a}(s)$ as the Laplacetransformed deflection and drive amplitude, respectively. $G_{0}(s)$ is the well-known transfer function of a harmonic oscillator. By partial fraction expansion, this transfer function can be rewritten in terms of the complex conjugate poles $p$ and $p^{*}$ of the denominator,

$$
\begin{aligned}
& G_{0}(s)=\frac{\omega_{0}^{2}}{p-p^{*}}\left(\frac{1}{s-p}-\frac{1}{s-p^{*}}\right) \\
& \text { with } p=-\omega_{\mathrm{c}}+i \sqrt{\omega_{0}^{2}-\omega_{\mathrm{c}}^{2}} \text { and } \omega_{\mathrm{c}}=\omega_{0} / 2 Q .
\end{aligned}
$$

Here, $\omega_{c}$ is introduced as a cutoff frequency, which is commonly known as the cantilever bandwidth. The transfer function written as eq. (29) is particularly useful, because it separates the second-order system into the equivalent of two parallel first-order systems. In the time-domain, the impulse response of each subsystem with a pole $p$ can readily be found by an inverse Laplace transform as $\exp (p t)$, which corresponds to an exponential decay $\exp \left(-\omega_{\mathrm{c}} t\right)$ of an oscillation excited at the resonance frequency $\omega_{\mathrm{r}}=\sqrt{\omega_{0}^{2}-\omega_{\mathrm{c}}^{2}}$. The impulse and step responses following eq. 29) are illustrated in fig. 2 . 

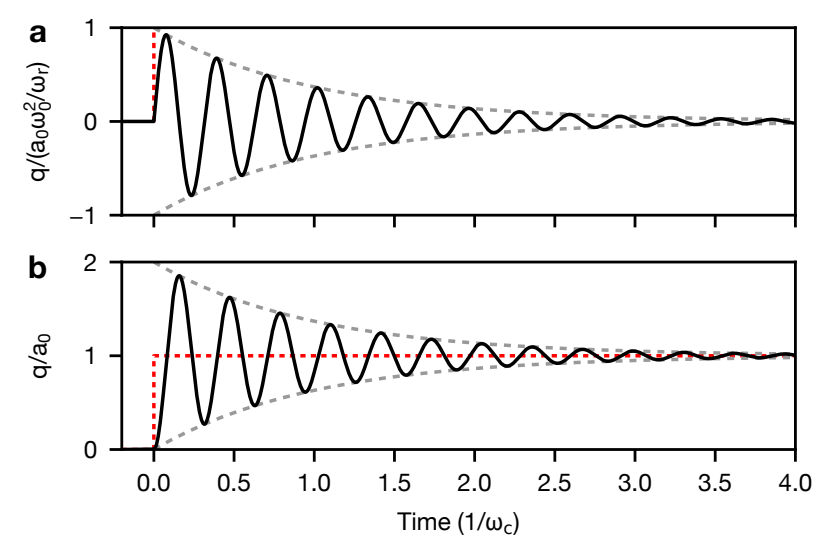

FIG. 2. Response of the deflection $q(t)$ to an impulse (a) and step (b) of the excitation $a(t)$ at $t=0$ for a harmonic oscillator with $\omega_{0}=2 \pi 100 \mathrm{kHz}$ and $Q=10$. The gray dashed lines indicate the envelope of the decaying oscillations at the resonance frequency $\omega_{\mathrm{r}} \approx \omega_{0}$.

Near the surface, with eqs. (2) and (25), the cantilever behavior around the steady-state solution is

$$
\begin{array}{r}
\ddot{q}+\omega_{0}\left(\frac{1}{Q}+\frac{\omega_{0}}{k}\left\langle\gamma_{\mathrm{ts}}\right\rangle\right) \dot{q}+\omega_{0}^{2}\left(1-\frac{\left\langle k_{\mathrm{ts}}\right\rangle}{k}\right) q \\
=\omega_{0}^{2} a(t)+\frac{\omega_{0}^{2}}{k} \delta F_{\text {int }}(t) .
\end{array}
$$

$\delta F_{\text {int }}(\mathrm{t})$ describes deviations of the tip-sample force from the steady state. In general, $\delta F_{\text {int }}$ follows from the Fourier series of $F_{\mathrm{ts}}$, eq. (5), as

$$
\delta F_{\text {int }}(t)=\sum_{n=-\infty}^{\infty} \delta \hat{f}_{n}(t) e^{i n \omega t}
$$

and the corresponding Laplace transform is

$$
\delta \tilde{F}_{\mathrm{int}}(s)=\sum_{n=-\infty}^{\infty} \delta \tilde{f}_{n}(s-i n \omega)
$$

where $\delta \tilde{f}_{n}(s)$ corresponds to the Laplace transform of the time-varying Fourier coefficient $\delta \hat{f}_{n}(t)$.

Laplace transform of eq. (31) results in

$$
\tilde{q}(s)=G(s)\left(\tilde{a}(s)+\delta \tilde{F}_{\mathrm{int}}(s) / k\right),
$$

where $G(s)$ is the steady-state transfer function of the cantilever, found by replacing $\omega_{0}^{2}$ and $1 / Q$ in the denominator of $G_{0}(s)$ according to eqs. (14) and (15). Accordingly, due to the interaction with the surface, the cantilever bandwidth and poles of $G(s)$ are changed to

$$
\begin{aligned}
\omega_{\mathrm{c}} & \rightarrow \omega_{\mathrm{c}}^{\prime}=\omega_{\mathrm{c}}+\left\langle\gamma_{\mathrm{ts}}\right\rangle \omega_{0}^{2} / 2 k \\
p & \rightarrow p^{\prime}=-\omega_{\mathrm{c}}^{\prime}+i \sqrt{\omega_{0}^{2}\left(1-\left\langle k_{\mathrm{ts}}\right\rangle / k\right)-\omega_{\mathrm{c}}^{\prime 2}} .
\end{aligned}
$$

In general, we must also account for amplitude and phase changes of the excitation signal introduced in eq. (4):

$$
a(t)=\left(a_{0}+\delta a_{\mathrm{exc}}(t)\right) \cos \left(\omega t+\delta \varphi_{\mathrm{exc}}(t)\right),
$$

where $\delta a_{\text {exc }}(t)$ and $\delta \varphi_{\text {exc }}(t)$ denote a small amplitude and phase modulation, respectively, which we add here to find the effect of changes to the excitation over time. Assuming that $\delta \varphi_{\text {exc }}(t) \ll 1$, the Laplace transform of eq. (37) can be written as

$$
\begin{aligned}
\tilde{a}(s)= & \tilde{a}_{\mathrm{s}}(s)+\delta \tilde{a}(s) \\
= & \frac{a_{0}}{2}\left(\frac{1}{s-i \omega}+\frac{1}{s+i \omega}\right) \\
& +\frac{1}{2}\left(\delta \tilde{a}_{\mathrm{exc}}(s-i \omega)+\delta \tilde{a}_{\mathrm{exc}}(s+i \omega)\right) \\
& +\frac{a_{0}}{2}\left(i \delta \tilde{\varphi}_{\mathrm{exc}}(s-i \omega)-i \delta \tilde{\varphi}_{\mathrm{exc}}(s+i \omega)\right),
\end{aligned}
$$

where $\tilde{a}_{\mathrm{s}}$ denotes the steady-state excitation, and $\delta \tilde{a}$ is a perturbation of the excitation due to amplitude and phase modulation.

The solution $\tilde{q}(s)$ of eq. (34) can be separated into the known solution of the steady state, $\tilde{q}_{\mathrm{s}}(s)$, and perturbations due to excitation $\delta \tilde{q}_{\text {exc }}(s)$ and interaction $\delta \tilde{q}_{\text {int }}(s)$ :

$$
\begin{aligned}
\tilde{q}(s) & =\tilde{q}_{\mathrm{s}}(s)+\delta \tilde{q}_{\mathrm{exc}}(s)+\delta \tilde{q}_{\mathrm{int}}(s) \\
\text { with } \quad \tilde{q}_{\mathrm{s}}(s) & =G(s) \tilde{a}_{\mathrm{s}}(s), \\
\delta \tilde{q}_{\mathrm{exc}}(s) & =G(s) \delta \tilde{a}(s), \text { and } \\
\delta \tilde{q}_{\mathrm{int}}(s) & =G(s) \delta \tilde{F}_{\mathrm{int}}(s) / k
\end{aligned}
$$

The steady-state perturbation of the deflection can be calculated explicitly. Note that $\tilde{a}_{\mathrm{s}}$ excites two oscillations at $\pm \omega$. The complex amplitudes of these oscillations are calculated using the final value theorem:

$$
\hat{q}_{\mathrm{s}}^{ \pm}:=\lim _{s \rightarrow 0} s \tilde{q}_{\mathrm{s}}(s \pm i \omega)=G( \pm i \omega) \frac{a_{0}}{2} .
$$

Because the cantilever is driven by a real signal, its steady-state response $q_{\mathrm{s}}(t)$ must be real as well. $\hat{q}_{\mathrm{s}}^{+}$and $\hat{q}_{\mathrm{s}}^{-}$must be complex conjugates:

$$
\hat{q}_{\mathrm{s}}^{+}=: \hat{q}_{\mathrm{s}} / 2 \quad \text { and } \quad \hat{q}_{\mathrm{s}}^{-}=\hat{q}_{\mathrm{s}}^{*} / 2 .
$$

The steady-state contribution to the deflection is thus

$$
\tilde{q}_{\mathrm{s}}(s)=\frac{\hat{q}_{\mathrm{s}} / 2}{s-i \omega}+\frac{\hat{q}_{\mathrm{s}}^{*} / 2}{s+i \omega} .
$$

The perturbation of $\tilde{q}(s)$ due to the excitation is

$$
\begin{aligned}
\delta \tilde{\mathrm{q}}_{\mathrm{exc}}(s)= & \frac{1}{2} G(s)\left[\delta \tilde{a}_{\mathrm{exc}}(s-i \omega)+\delta \tilde{a}_{\mathrm{exc}}(s+i \omega)\right] \\
& +\frac{i a_{0}}{2} G(s)\left[\delta \tilde{\varphi}_{\mathrm{exc}}(s-i \omega)-\delta \tilde{\varphi}_{\mathrm{exc}}(s+i \omega)\right] .
\end{aligned}
$$


As a result of eqs. 111 and 13, the dynamics of the zero- and first-order Fourier components of the transient interaction force $\delta F_{\text {int }}$ can be interpreted as perturbations of the tip-sample force and complex force gradient, respectively:

$$
\begin{aligned}
\delta \tilde{f}_{0}(s) & =\delta \tilde{F}_{\mathrm{ts}}(s) \\
\delta \tilde{f}_{ \pm 1}(s) & =\delta \tilde{k}_{ \pm 1}(s) \hat{q}_{\mathrm{s}}^{ \pm}=\left[\delta \tilde{k}_{\mathrm{ts}}(s) \mp i \omega \tilde{\gamma}_{\mathrm{ts}}(s)\right] \hat{q}_{\mathrm{s}}^{ \pm} .
\end{aligned}
$$

Separating $\hat{f}_{0}$ and $\hat{f}_{ \pm 1}$ from higher harmonics, the transient interaction force, eq. (33), can be written as

$$
\begin{aligned}
\delta F_{\text {int }}(s)= & \delta \tilde{f}_{0}(s)+\delta \tilde{f}_{1}(s-i \omega)+\delta \tilde{f}_{-1}(s+i \omega) \\
& +\sum_{n=2}^{\infty}\left[\delta \tilde{f}_{n}(s-i n \omega)+\delta \tilde{f}_{-n}(s+i n \omega)\right]
\end{aligned}
$$

With eq. 40d the resulting perturbation of the deflection $\delta \tilde{q}_{\text {int }}$ is therefore

$$
\begin{aligned}
\delta \tilde{q}_{\mathrm{int}}(s)= & \frac{1}{k} G(s)\left\{\delta \tilde{F}_{\mathrm{ts}}(s)\right. \\
& +\frac{1}{2}\left[\delta \tilde{k}_{\mathrm{ts}}(s-i \omega)-i \omega \delta \tilde{\gamma}_{\mathrm{ts}}(s-i \omega)\right] \hat{q}_{\mathrm{s}} \\
& +\frac{1}{2}\left[\delta \tilde{k}_{\mathrm{ts}}(s+i \omega)+i \omega \delta \tilde{\gamma}_{\mathrm{ts}}(s+i \omega)\right] \hat{q}_{\mathrm{s}}^{*} \\
& \left.+\sum_{n=2}^{\infty}\left[\delta \tilde{f}_{n}(s-i n \omega)+\delta \tilde{f}_{-n}(s+i n \omega)\right]\right\} .
\end{aligned}
$$

This equation describes the behavior of the cantilever for arbitrarily chosen perturbations of the force $\delta \tilde{F}_{\mathrm{ts}}$, force gradient $\delta \tilde{k}_{\mathrm{ts}}$, damping coefficient $\delta \tilde{\gamma}_{\mathrm{ts}}$, or higher harmonics of the interaction force, given only the approximation that the cantilever oscillation remains harmonic at all times. Perturbations of the tip-sample force $\delta \tilde{F}_{\text {ts }}$ drive the cantilever directly via its steady-state transfer function $G(s)$, whereas perturbations of the force gradient $\delta \tilde{k}_{\mathrm{ts}}$ or damping coefficient $\delta \tilde{\gamma}_{\mathrm{ts}}$ are shifted up and down in the frequency domain by the drive frequency $\omega$ before they enter $G(s)$. Similarly, contributions from the $n$-th harmonic of the interaction force are frequencyshifted by $\pm n \omega$.

\section{BASEBAND SIGNAL}

The results of the previous section can be used to derive the dynamics of the baseband signal, i.e., the signal detected by a lock-in amplifier at the excitation frequency $\omega$ and phase $\delta \varphi_{\text {exc }}$. In the time domain, the baseband signal is given by

$$
\begin{aligned}
q_{\mathrm{b}}(t) & =2 h(t) * q_{\omega}(t) \\
& \approx 2 h(t) *\left[\left(1-i \delta \varphi_{\mathrm{exc}}(t)\right) e^{-i \omega t} q(t)\right]
\end{aligned}
$$

where

$$
q_{\omega}(t)=\exp \left[-i \omega t-i \delta \varphi_{\mathrm{exc}}(t)\right] q(t)
$$

denotes the down-conversion with respect to the excitation at $\omega$, the prefactor 2 is chosen to obtain peak amplitudes, $h(t)$ is the impulse response of a low-pass filter, and the asterisk $*$ denotes a convolution. In the following, we choose $h(t)$ to filter frequencies beyond the modulation frequency $\omega$.

There are several ways to find $q(t)$ and $q_{\omega}(t)$ as required for the solution. For example, $q(t)$ can be obtained directly by solving the equation of motion, eq. (2), e.g., by Verlet 36] integration or Runge-Kutta methods. Because we are interested in the demodulated deflection $q_{\omega}(t)$, we can make use of eq. 29, which describes the separation of the second-order system into two first-order systems. After down-conversion, $\tilde{q}_{\omega}(s)=q(s+i \omega)$, one system describes the dynamics at the baseband, while the other system contains high-frequency oscillations at $\approx \omega_{0}+\omega$ which are removed by the low-pass filter.

With these considerations, the equation of motion for the baseband deflection $q_{\mathrm{b}}(t)$ is obtained by an inverse Laplace transform of eq. 29) as

$$
\dot{q}_{\mathrm{b}}-(p-i \omega) q_{\mathrm{b}}=\frac{\omega_{0}^{2}}{p-p^{*}} a_{\mathrm{b}}(t),
$$

where $a_{\mathrm{b}}(t)$ is the baseband excitation signal. The poles $p$ and $p^{*}$, given by eq. (36), include the interaction of the tip with the surface through the averages $\left\langle k_{\mathrm{ts}}\right\rangle$ and $\left\langle\gamma_{\mathrm{ts}}\right\rangle$.

Next, we derive the baseband dynamics in the frequency domain. Analytical expressions and transfer functions are necessary for an intuitive understanding of the cantilever behavior and for the design of feedback loops. Using the Laplace transform of eq. (50), we obtain

$$
\begin{aligned}
\tilde{q}_{\mathrm{b}}(s) & \approx 2 H(s)\left[\tilde{q}(s+i \omega)-i \delta \tilde{\varphi}_{\mathrm{exc}}(s) * \tilde{q}(s+i \omega)\right] \\
& \approx H(s)\left[2 \tilde{q}(s+i \omega)-i \delta \tilde{\varphi}_{\mathrm{exc}}(s) \hat{q}_{\mathrm{s}}\right]
\end{aligned}
$$

where $H(s)$ indicates the transfer function of the lowpass filter. In the latter step we use $\tilde{q} \approx \tilde{q}_{\mathrm{s}}$ and assume that $\delta \tilde{\varphi}$ vanishes for frequencies above $\omega$.

In the following, we indicate filtered quantities by a prime, e.g., $\tilde{q}^{\prime}(s)=H(s) \tilde{q}(s)$. The filtered contributions to the deflection are

$$
\begin{aligned}
2 \tilde{q}_{\mathrm{s}}^{\prime}(s+i \omega)= & \hat{q}_{\mathrm{s}} / s \\
2 \delta \tilde{q}_{\mathrm{int}}^{\prime}(s+i \omega)= & \frac{1}{k} G(s+i \omega)\left\{\left[\delta \tilde{k}_{\mathrm{ts}}(s)-i \omega \delta \tilde{\gamma}_{\mathrm{ts}}(s)\right] \hat{q}_{\mathrm{s}}\right. \\
& +\left[\delta \tilde{k}_{\mathrm{ts}}(s+2 i \omega)+i \omega \delta \tilde{\gamma}_{\mathrm{ts}}(s+2 i \omega)\right] \hat{q}_{\mathrm{s}}^{*} \\
& \left.+2 \delta \tilde{F}_{\mathrm{ts}}(s+i \omega)\right\}, \text { and } \\
2 \delta \tilde{q}_{\mathrm{exc}}^{\prime}(s+i \omega)= & G(s+i \omega)\left[\delta \tilde{a}_{\mathrm{exc}}(s)+\delta \tilde{\varphi}_{\mathrm{exc}}(s) i a_{0}\right] .
\end{aligned}
$$


For clarity of the presentation, higher harmonic terms of $\delta \tilde{q}_{\text {int }}$ are left out in $\delta \tilde{q}_{\text {int }}^{\prime}$, because they are typically negligible if they are not driven externally at a frequency of $(n \pm 1) \omega$.

Inserting eqs. (55a) to 55c into eq. (54), we obtain the behaviour of the baseband deflection

$$
\begin{aligned}
\tilde{q}_{\mathrm{b}}^{\prime}(s)= & \left\{\frac{1}{s}+\frac{1}{k} G(s+i \omega)\left[\delta \tilde{k}_{\mathrm{ts}}(s)-i \omega \delta \tilde{\gamma}_{\mathrm{ts}}(s)\right]\right\} \hat{q}_{\mathrm{s}} \\
+ & \frac{1}{k} G(s+i \omega)\left[\delta \tilde{k}_{\mathrm{ts}}(s+2 i \omega)-i \omega \delta \tilde{\gamma}_{\mathrm{ts}}(s+2 i \omega)\right] \hat{q}_{\mathrm{s}}^{*} \\
& +\frac{1}{k} G(s+i \omega)\left[\delta \tilde{F}_{\mathrm{ts}}(s+i \omega)+k \delta \tilde{a}_{\mathrm{exc}}(s)\right] \\
& +i\left[\frac{G(s+i \omega)}{G(i \omega)}-1\right] \delta \tilde{\varphi}_{\mathrm{exc}}(s) \hat{q}_{\mathrm{s}}
\end{aligned}
$$

The resulting deflection can be viewed as a combined amplitude and phase modulation

$$
q(t)=q_{\mathrm{s}}[1+\delta m(t)] \cos \left(\omega t+\varphi_{\mathrm{s}}+\delta \varphi(t)\right),
$$

where $q_{\mathrm{s}}=\left|\hat{q}_{\mathrm{s}}\right|$ and $\varphi_{\mathrm{s}}=\arg \hat{q}_{\mathrm{s}}$. For small modulations $\delta m$ and $\delta \varphi$ (narrowband approximation), the corresponding baseband signal is

$$
q_{\mathrm{b}}^{\prime}(s)=\left\{\frac{1}{s}+\delta \tilde{m}(s)+i \delta \tilde{\varphi}(s)\right\} \hat{q}_{\mathrm{s}} .
$$

We can furthermore introduce the complex modulation signal $\delta \tilde{\alpha}:=\delta \tilde{m}+i \delta \tilde{\varphi}$, which captures the amplitude and phase modulation in its real and imaginary component, respectively. Comparing eq. (58) and eq. (56), we obtain

$$
\begin{aligned}
\delta \tilde{\alpha}(s)= & \frac{1}{k} G(s+i \omega)\left[\delta \tilde{k}_{\mathrm{ts}}(s)-i \omega \delta \tilde{\gamma}_{\mathrm{ts}}(s)\right] \\
+ & \frac{1}{k} \frac{\hat{q}_{\mathrm{s}}^{*}}{\hat{q}_{\mathrm{s}}} G(s+i \omega)\left[\delta \tilde{k}_{\mathrm{ts}}(s+2 i \omega)+i \omega \delta \tilde{\gamma}_{\mathrm{ts}}(s+2 i \omega)\right] \\
& +\frac{1}{k a_{0}} \frac{G(s+i \omega)}{G(i \omega)}\left[\delta \tilde{F}_{\mathrm{ts}}(s+i \omega)+k \delta \tilde{a}_{\mathrm{exc}}(s)\right] \\
& +i\left[\frac{G(s+i \omega)}{G(i \omega)}-1\right] \delta \tilde{\varphi}_{\mathrm{exc}}(s) .
\end{aligned}
$$

We can further simplify eq. 59 if we assume an excitation at the eigenfrequency $\omega_{0}$. Then, the cantilever response is phase-lagging at $90^{\circ}$ and amplified by $Q$, $G(i \omega) \approx G\left(i \omega_{0}\right)=-i Q$, and $\hat{q}_{\mathrm{s}}^{*} / \hat{q}_{\mathrm{s}} \approx-1$. The steadystate transfer function of the cantilever $G(s)$ can be approximated by a first-order system:

$$
G(s+i \omega) \approx \frac{G(i \omega)}{1+s / \omega_{\mathrm{c}}} \approx-\frac{i}{2} \frac{\omega_{0}}{\omega_{\mathrm{c}}+s} .
$$

In this limit, we obtain

$$
\begin{aligned}
\delta \tilde{\alpha}(s) \approx & \frac{1}{k a_{0}} \frac{\omega_{\mathrm{c}}}{\omega_{\mathrm{c}}+s}\left[\delta \tilde{F}_{\mathrm{ts}}(s+i \omega)+k \delta \tilde{a}_{\mathrm{exc}}(s)\right] \\
& -\frac{\omega_{0}}{2 k} \frac{1}{\omega_{\mathrm{c}}+s}\left[i \delta \tilde{k}_{\mathrm{ts}}(s)+\omega \delta \tilde{\gamma}_{\mathrm{ts}}(s)\right] \\
& +\frac{\omega_{0}}{2 k} \frac{1}{\omega_{\mathrm{c}}+s}\left[i \delta \tilde{k}_{\mathrm{ts}}(s+2 i \omega)-\omega \delta \tilde{\gamma}_{\mathrm{ts}}(s+2 i \omega)\right] \\
& -i \frac{s}{\omega_{\mathrm{c}}+s} \delta \tilde{\varphi}_{\mathrm{exc}}(s) .
\end{aligned}
$$

We can further split the frequency-shifted perturbations into components in- and out-of-phase with the drive signal,

$$
\begin{aligned}
\delta \tilde{F}_{\mathrm{ts}}(s+i \omega) & =\delta \tilde{F}_{\mathrm{ts}}^{\omega, \mathrm{i}}(s)+i \delta \tilde{F}_{\mathrm{ts}}^{\omega, \mathrm{q}}(s), \\
\delta \tilde{k}_{\mathrm{ts}}(s+2 i \omega) & =\delta \tilde{k}_{\mathrm{ts}}^{2 \omega, \mathrm{i}}(s)+i \delta \tilde{k}_{\mathrm{ts}}^{2 \omega, \mathrm{q}}(s), \\
\delta \tilde{\gamma}_{\mathrm{ts}}(s+2 i \omega) & =\delta \tilde{\gamma}_{\mathrm{ts}}^{2 \omega, \mathrm{i}}(s)+i \delta \tilde{\gamma}_{\mathrm{ts}}^{2 \omega, \mathrm{q}}(s) .
\end{aligned}
$$

For the components of the amplitude and phase modulation, we obtain:

$$
\begin{aligned}
\delta \tilde{m}(s) \approx & \frac{1}{\omega_{\mathrm{c}}+s}\left\{\frac{\omega_{\mathrm{c}}}{k a_{0}}\left[\delta \tilde{F}_{\mathrm{ts}}^{\omega, \mathrm{i}}(s)+k \delta \tilde{a}_{\mathrm{exc}}(s)\right]\right. \\
& \left.-\frac{\omega_{0}}{2 k}\left[\delta \tilde{k}_{\mathrm{ts}}^{2 \omega, \mathrm{q}}(s)+\omega \delta \tilde{\gamma}_{\mathrm{ts}}(s)+\omega \delta \tilde{\gamma}_{\mathrm{ts}}^{2 \omega, \mathrm{i}}(s)\right]\right\} \\
\delta \tilde{\varphi}(s) \approx & \frac{1}{\omega_{\mathrm{c}}+s}\left\{\frac{\omega_{\mathrm{c}}}{k a_{0}} \delta \tilde{F}_{\mathrm{ts}}^{\omega, \mathrm{q}}(s)\right. \\
& \left.-\frac{\omega_{0}}{2 k}\left[\delta \tilde{k}_{\mathrm{ts}}(s)-\delta \tilde{k}_{\mathrm{ts}}^{2 \omega, \mathrm{i}}(s)+\omega \delta \tilde{\gamma}_{\mathrm{ts}}^{2 \omega, \mathrm{q}}(s)\right]\right\} \\
& -\frac{s}{\omega_{\mathrm{c}}+s} \delta \tilde{\varphi}_{\mathrm{exc}}(s) .
\end{aligned}
$$

The instantaneous frequency is defined as the phase derivative. Therefore, the frequency modulation $\delta \tilde{\omega}$ resulting from the phase modulation in eq. $65 \mathrm{~b}$ can be derived as

$$
\begin{aligned}
\delta \tilde{\omega}(s)= & s \delta \tilde{\varphi}(s) \\
\approx & \frac{s}{\omega_{\mathrm{c}}+s}\left\{\frac{\omega_{\mathrm{c}}}{k a_{0}} \delta \tilde{F}_{\mathrm{ts}}^{\mathrm{q}}(s+i \omega)-\delta \tilde{\omega}_{\mathrm{exc}}(s)\right. \\
& \left.-\frac{\omega_{0}}{2 k}\left[\delta \tilde{k}_{\mathrm{ts}}(s)-\delta \tilde{k}_{\mathrm{ts}}^{2 \omega, \mathrm{i}}(s)+\omega \delta \tilde{\gamma}_{\mathrm{ts}}^{2 \omega, \mathrm{q}}(s)\right]\right\},
\end{aligned}
$$

where $\delta \tilde{\omega}_{\text {exc }}=s \delta \tilde{\varphi}_{\text {exc }}$ is the modulation of the drive frequency.

This allows for the following general observations:

- The amplitude is modulated by changes of the excitation amplitude $\delta \tilde{a}_{\text {exc }}$, the damping coefficient $\delta \tilde{\gamma}_{\text {ts }}$, and its in-phase component at $2 \omega$. The amplitude can also be modulated by the in-phase component of a tip-sample force $\delta \tilde{F}_{\mathrm{ts}}$ at $\omega$ and the quadrature component of $\delta \tilde{k}_{\mathrm{ts}}$ at $2 \omega$. These changes all pass through a lowpass filter at $\omega_{\mathrm{c}}$.

- The phase and instantaneous frequency are modulated due to changes of the force gradient $\delta \tilde{k}_{\mathrm{ts}}$, its in-phase component at $2 \omega$. It is also modulated by the quadrature component of $\delta \tilde{F}_{\text {ts }}$ at $\omega$ and the quadrature component of $\delta \tilde{\gamma}_{\text {ts }}$ at $2 \omega$. For the detected phase, these changes all pass through a lowpass filter, whereas the excitation phase enters via a highpass filter at $\omega_{\mathrm{c}}$. For the instantaneous frequency, there is a highpass filter at $\omega_{\mathrm{c}}$ common to all inputs. 
A widespread misconception, dating back to the time when the FM-AFM technique was first introduced by Albrecht et al. 4, is that phase and frequency changes propagate instantaneously. This statement is obviously true only to a limited extent. Especially in ambient or liquid environments, quality factors are typically well below 1000, and the cutoff frequency can be on the order of hundreds of Hertz. Phase changes due to interactions with the surface are then detected easily within the bandwidth $\omega_{\mathrm{c}}$, whereas changes of the excitation phase can cause strong transients due to the highpass characteristic. On the other hand, in high vacuum quality factors of $\sim 100000$ can be achieved, resulting in cutoff frequencies on the order of $1 \mathrm{~Hz}$. When slow processes are observed, or when feedback loops are intentionally kept at low speeds, the exact expression should be used also under vacuum conditions. Only for quality factors $Q$ approaching infinity, the cutoff frequency is negligible, and phase changes of the excitation affect the cantilever oscillation nearly immediately.

\section{TRANSFER FUNCTIONS FOR FM-AFM}

Next, we derive transfer functions relevant for FMAFM operation. Equation (59) contains the behavior of the amplitude and phase modulations as a function of the driving amplitude and phase of the cantilever. These are the transfer functions of amplitude and phase, $G_{\mathrm{m}}(s)$ and $G_{\varphi}(s)$, respectively,

$$
\begin{aligned}
G_{\mathrm{m}}(s) & :=\frac{\delta \tilde{m}(s)}{\delta \tilde{a}_{\mathrm{exc}}(s)}=\frac{1}{a_{0}} \frac{G(s+i \omega)}{G(i \omega)} \approx \frac{1}{a_{0}} \frac{\omega_{\mathrm{c}}}{\omega_{\mathrm{c}}+s} \\
G_{\varphi}(s) & :=\frac{\delta \tilde{\varphi}(s)}{\delta \tilde{\omega}_{\mathrm{exc}}(s)}=\frac{1}{s}\left[\frac{G(s+i \omega)}{G(i \omega)}-1\right] \approx-\frac{1}{\omega_{\mathrm{c}}+s} .
\end{aligned}
$$

The approximations following from eqs. (65a) and (65b) are valid for an excitation close to $\omega_{0}$ and assume that $\omega_{\mathrm{c}} \ll \omega_{0}$, such that the cantilever transfer function can be considered a first-order system.

As indicated by eq. 67), amplitude changes propagate via a low-pass filter at $\omega_{\mathrm{c}}$. Changes of the drive frequency propagate via eq. $(68)$ to the detected cantilever phase, which has the characteristics of an integrator with a cutoff frequency $\omega_{\mathrm{c}}$. As discussed below, this behaviour has important implications for the design of a phase-locked loop.

In the following, we use eqs. 67) and 68 to find the closed-loop behaviour of the feedback loops governing FM-AFM performance and stability.

\section{A. Phase-locked loop}

A PLL is used to track the resonance frequency and to excite the cantilever accordingly 37-39. The output signal of the PLL is the frequency shift $\Delta \omega$, which is used as

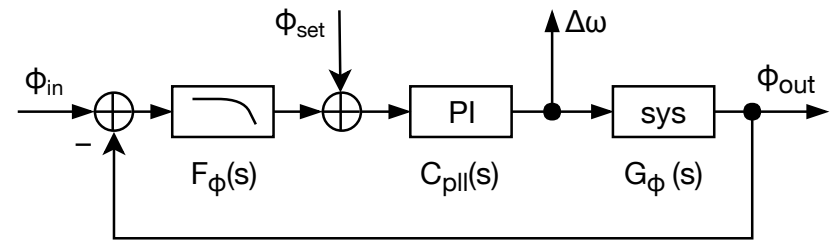

FIG. 3. Block diagram of the phase-locked loop.

the input signal to the topography feedback loop. Following eq. 68 every change of the instantaneous resonance frequency has an effect on the detected phase. Therefore, by adjusting the excitation frequency to maintain a constant phase, the cantilever can be driven at resonance.

A PLL is composed of several building blocks: a tunable reference oscillator, a phase detector to measure the phase relation of the input oscillation to the reference, and a loop filter responsible for closed-loop control.

The phase detector is commonly built by downconversion of the input signal by the reference oscillator, followed by low-pass filter to remove high-frequency mixing products. To achieve higher bandwidths up to the cantilever eigenfrequency, alternative phase detectors have been proposed recently 40,42 .

For small phase shifts, the phase detector can be approximated by the transfer function of the filter $F_{\varphi}(s)$. It should be mentioned that the phase detector is no longer linear for the large phase shifts which appear initially when acquiring the phase lock [43. Next, we therefore examine the performance in the phase-locked state only.

The block diagram in fig. 3 illustrates the complete phase feedback loop. To maintain the phase at resonance (setpoint $\varphi_{\text {set }}=-90^{\circ}$ ), the drive frequency is adjusted by a proportional-integral (PI) controller, $C_{\text {pll }}$. Phase changes of the cantilever, which describe the deviations from resonance, enter the system via the input phase $\varphi_{\text {in }}$, whereas the resulting controlled phase is given by $\varphi_{\text {out }}$. The closed-loop transfer functions of the PLL are:

$$
\begin{aligned}
\tilde{\varphi}_{\text {out }} & =G_{\varphi} C_{\mathrm{pll}}\left[\varphi_{\text {set }}+F_{\varphi}\left(\tilde{\varphi}_{\text {in }}-\tilde{\varphi}_{\text {out }}\right)\right] \\
\Rightarrow \frac{\tilde{\varphi}_{\text {out }}}{\tilde{\varphi}_{\text {in }}} & =\frac{G_{\varphi} C_{\mathrm{pll}} F_{\varphi}}{1+G_{\varphi} C_{\mathrm{pll}} F_{\varphi}}, \frac{\tilde{\varphi}_{\text {out }}}{\tilde{\varphi}_{\text {set }}}=\frac{G_{\varphi} C_{\mathrm{pll}}}{1+G_{\varphi} C_{\mathrm{pll}} F_{\varphi}} .
\end{aligned}
$$

Note that the transfer functions for $\tilde{\varphi}_{\text {in }}$ and $\tilde{\varphi}_{\text {set }}$ are slightly different, because changes of the setpoint are not affected by the phase detection filter $F_{\varphi}$. Care should be taken when testing the response of the feedback loop experimentally by changing the setpoint, since there might be deviations from the closed-loop response rejecting disturbances $\varphi_{\text {in }}$.

The dynamic performance of the feedback loop is determined by the poles of the PLL transfer function, which are the zeros of $1+G_{\mathrm{ol}}$, where $G_{\mathrm{ol}}=G_{\varphi} C_{\mathrm{pll}} F_{\varphi}$ is the open-loop transfer function.

For $C_{\text {pll }}(s)$, we can write

$$
C_{\mathrm{pll}}(s)=K_{\mathrm{p}}+\frac{K_{\mathrm{i}}}{s}=K_{\mathrm{p}} \frac{\omega_{\mathrm{pi}}+s}{s},
$$


where $K_{\mathrm{p}}$ and $K_{\mathrm{i}}$ are the proportional and integral gains of the feedback loop, and $\omega_{\mathrm{pi}}=K_{\mathrm{i}} / K_{\mathrm{p}}$. Therefore, in the open-loop transfer function $G_{\mathrm{ol}}$ the pole at $\omega_{\mathrm{c}}$ due to eq. (68) can be cancelled by the zero at $\omega_{\mathrm{pi}}$ due to eq. 70 by choosing appropriate feedback parameters.

Furthermore, the poles and zeros of the filter $F_{\varphi}$ can be chosen such that they are well above the desired closedloop bandwidth. In this limit, the closed-loop transfer function of the PLL is the response of a first-order lowpass filter

$$
\frac{\tilde{\varphi}_{\mathrm{out}}}{\tilde{\varphi}_{\mathrm{in}}} \approx \frac{\omega_{\mathrm{cl}}}{s+\omega_{\mathrm{cl}}}
$$

where the closed-loop bandwidth is $\omega_{\mathrm{cl}}=-K_{\mathrm{p}}$. Note that the proportional gain $K_{\mathrm{p}}$ must be negative to obtain a stable closed-loop system under negative feedback. This is because following eq. (68) an increased drive frequency results in a reduced phase response.

PLL transfer functions in literature [39, 44, are commonly derived under the assumption of negligible damping, i.e., $\omega_{\mathrm{c}} \rightarrow 0$. The detected phase in eq. (68) is then merely an integral of frequency, and a first-order PLL defined by its proportional gain alone would suffice for good performance. Although in ultra-high vacuum this assumption holds very well [39, with lower quality factors as typical in ambient or liquid environments there can be significant deviations which can cause experimental artifacts [45, 46].

Note that most textbooks 43, 47, 48, deal with PLLs in the context of signals and communications systems, where the detected phase is indeed the integrated change of the reference frequency. In the context of AFM this situation is found in combination with a self-excitation setup [4, in which the detected deflection of the cantilever is delayed or phase-shifted, amplified, and applied as an excitation signal. For this configuration, a proportional-type controller is sufficient, because the measured frequency shift is detected only and not fed back via the excitation signal.

For PLL-based excitation, in contrast, the cantilever transfer function enters the excitation loop and must be considered. The main advantage of PLL-based excitation is the clean driving signal derived from the narrow-band phase detector, which enables working with low-Q cantilevers [38] and provides better noise performance [44]. It is unfortunate that several publications focusing on FM-AFM instrumentation and simulation initially neglected the integral feedback required in the general case [39, 49, 50. Related to that, the phase response of the cantilever must necessarily be considered within the phase feedback loop. Attempts to incorporate the phase response otherwise, e.g., from an outer feedback loop [38, or treating the combined system as interlaced control loops [51] have resulted in contradictory transfer functions.

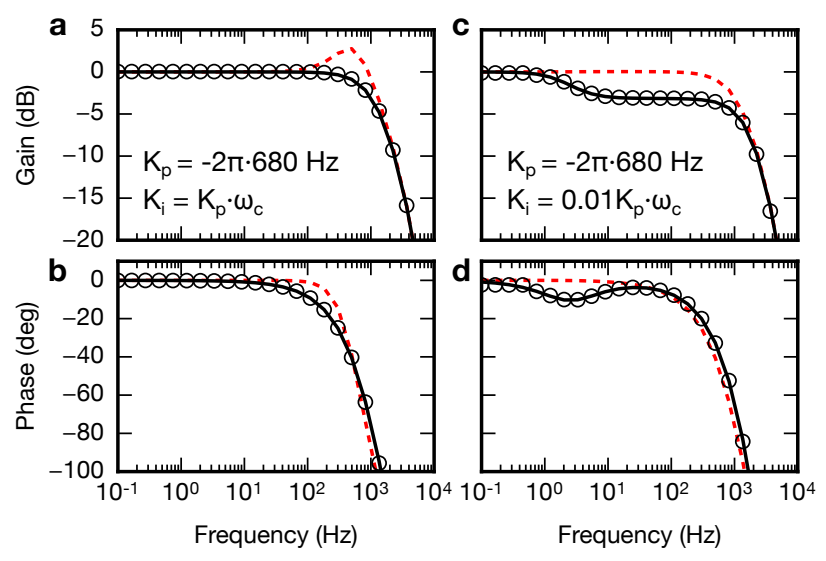

FIG. 4. Influence of the integral gain on the closed-loop behaviour of the PLL. The black lines indicate the response as calculated from eq. 69. The circles show a full numerical simulation based on the cantilever dynamics and detection system. The response for an ideal oscillator, i.e., $\omega_{\mathrm{c}}=0$, is indicated by a dashed red line. A cantilever with $\omega_{0}=$ $2 \pi 300 \mathrm{kHz}$ and $Q=500$ is assumed, and the phase detector is modeled as a four-pole lowpass with a $-3 \mathrm{~dB}$ bandwidth of $4 \mathrm{kHz}$. (a) Gain and (b) phase response for a PLL tuned to a bandwidth of $1 \mathrm{kHz}$. (c) Gain and (d) phase response with integral gain reduced by a factor of 100 .

To estimate the error with a proportional-only controller $\left(C_{\mathrm{pll}}=K_{\mathrm{p}}\right)$, we derive the closed-loop transfer function, omitting the phase detector, as

$$
\frac{\tilde{\varphi}_{\text {out }}}{\tilde{\varphi}_{\text {in }}} \approx \frac{1}{1-\omega_{\mathrm{c}} / K_{\mathrm{p}}} \frac{\omega_{\mathrm{c}}-K_{\mathrm{p}}}{s+\omega_{\mathrm{c}}-K_{\mathrm{p}}},
$$

corresponding to a lowpass filter with the cutoff frequency $\omega_{\mathrm{c}}-K_{\mathrm{p}}$ and gain reduced by a factor of $(1-$ $\left.\omega_{\mathrm{c}} / K_{\mathrm{p}}\right)$. If $\omega_{\mathrm{c}}$ is not negligible compared to $K_{\mathrm{p}}$, a proportional-only controller can lead to an apparent reduction of the frequency shift $\Delta \omega$ and thereby cause inaccurate FM-AFM measurements.

Figure 4 shows the influence of the integral gain on the closed-loop behaviour of the PLL for a real $\left(\omega_{0}=\right.$ $2 \pi 300 \mathrm{kHz}, Q=500)$ and ideal oscillator $\left(\omega_{\mathrm{c}}=0\right)$. Also shown are the results of a simulation of the cantilever dynamics and the detection system. For the simulation, the time is discretized to $\Delta t=(1 \mathrm{MHz})^{-1}$, and the equation of motion, eq. (31), is solved by velocity Verlet integration with 10 intermediate steps. At each time step $\Delta t$, the phase is detected by a lock-in amplifier and maintained at $-90^{\circ}$ by a PI controller. To obtain each point in the simulation, the resonance frequency is modulated at a single frequency $\omega_{\mathrm{m}}$, and the resulting frequency shift signal is recorded during at least ten cycles. Gain and phase are calculated from the Fourier coefficient of the frequency shift signal at $\omega_{\mathrm{m}}$.

The simulation shows that the closed-loop behaviour follows eq. (69) very well. As described above, the closedloop bandwidth scales with $K_{\mathrm{p}}$. A hundredfold reduction 
of the integral gain results in a reduction of the amplitude at higher frequencies by nearly $30 \%$ due to dominant proportional-type action. In an actual experiment, a controller tuned similar to fig. 4(c) and (d) can be detrimental. To the operator, the feedback loop appears to work properly, because there is no static error and the frequency shift $\Delta \omega$ appears to follow up to the desired bandwidth. It is easiest to detect such a situation experimentally from a step response, for example by retracting the tip from the surface. With too little integral action, $\Delta \omega$ would slowly creep to the final value.

\section{B. Amplitude controller}

Detected amplitude changes $\delta \tilde{A}$ are determined by the modulation $\delta \tilde{m}$ and the steady-state cantilever amplitude $A=\left|\hat{q}_{\mathrm{s}}\right|$,

$$
G_{\mathrm{A}}(s)=\frac{\delta \tilde{m}(s) A}{\delta \tilde{a}_{\mathrm{exc}}(s)}=G_{\mathrm{m}}(s) A \approx Q \frac{\omega_{\mathrm{c}}}{\omega_{\mathrm{c}}+s},
$$

where the approximation holds for excitation at resonance. Similar to the derivation of the PLL transfer function, the closed-loop transfer function of the amplitude controller is obtained as

$$
\frac{\tilde{A}_{\mathrm{out}}}{\tilde{A}_{\mathrm{in}}}=\frac{G_{\mathrm{A}} C_{\mathrm{A}} F_{\mathrm{A}}}{1+G_{\mathrm{A}} C_{\mathrm{A}} F_{\mathrm{A}}},
$$

where $F_{\mathrm{A}}(s)$ is the transfer function of the amplitude detection filter and a PI controller $C_{\mathrm{A}}(s)$ is used to maintain a constant amplitude. Choosing $\omega_{\text {pi }}$ again to compensate the dominating pole at $\omega_{\mathrm{c}}$, and neglecting the filter $F_{\mathrm{A}}$, we obtain

$$
\frac{\tilde{A}_{\mathrm{out}}}{\tilde{A}_{\mathrm{in}}} \approx \frac{\omega_{\mathrm{cl}}}{s+\omega_{\mathrm{cl}}} \quad \text { with } \quad \omega_{\mathrm{cl}}=K_{\mathrm{p}} Q \omega_{\mathrm{c}}=K_{\mathrm{p}} \omega_{0} / 2 .
$$

\section{DISCUSSION}

The relations derived in sections IV and V together with the steady-state solution from section III describe the full input/output relationship of interaction and driving forces acting on the deflection of the AFM tip.

The solution for perturbations of the deflection $\delta \tilde{q}_{\text {int }}$, eq. 48 , shows that changes of the interaction $\delta \tilde{F}_{\text {ts }}$ enter at low frequencies without change, because the transfer function $G(s)$ at low frequencies is unity. Particularly, there is no additional lowpass filtering, even if the common designation cantilever bandwidth for $\omega_{\mathrm{c}}$ would suggest so. Modulation at the drive frequency $\omega$ in $\delta \tilde{k}_{\mathrm{ts}}$ or $\delta \tilde{\gamma}_{\text {ts }}$ can also be detected immediately in the deflection signal, that is, without prior demodulation.

The complex baseband signal, eq. 59, describes the cantilever response at the driving frequency $\omega$. In our derivation, we consider weak perturbations around the steady-state solution. Besides, there are no assumptions about the quality factor of the cantilever or the deviation of the drive frequency from resonance. Hence, this solution is applicable even in environments of high damping.

Equations 65a), 65b and (66) describe the amplitude, phase, and frequency response in an environment of moderate damping when driving close to resonance. In particular, these equations reflect the well-known fact that the phase and frequency modulation of the cantilever is primarily due to a modulation of the force gradient, whereas the amplitude is modulated due to dissipation. Moreover, amplitude and phase are modulated by the in-phase and quadrature components of the driving force, respectively.

In addition, in-phase and quadrature components of $\delta \tilde{k}_{\text {ts }}$ or $\delta \tilde{\gamma}_{\text {ts }}$ at $2 \omega$ also modulate the amplitude and phase of the deflection at $\omega$. Such heterodyne modulation and detection offers an interesting route to probe tip-sample interactions at high frequencies. The effect has been utilized, for example, to implement a force gradient sensitive detection method for Kelvin probe force microscopy using the dissipation channel [52. Because the dissipation channel, contained in $\delta m$, is orthogonal to the frequency modulation $\delta \omega$, a larger Kelvin detection bandwidth can be achieved compared to the traditional approach of modulating $\delta k_{\mathrm{ts}}$ at low frequencies. A downside to this approach is that amplitude changes due to increased real dissipation or changes of the driving behaviour may be mistaken for changes of the measured surface potential.

Higher harmonic terms in eq. (48) appear due to the highly non-linear tip-sample force even if the tip oscillation can be considered harmonic. In practice, they are difficult to detect off-resonance at frequencies $n \omega$. However, a modulation of the tip-sample force at a frequency $\omega_{\mathrm{m}}$ also modulates the harmonics $\delta \tilde{f}_{ \pm n}$ at $\omega_{\mathrm{m}}$. Frequency mixing with harmonics of the carrier oscillation can therefore be used to excite the cantilever at frequencies $n \omega+\omega_{\mathrm{m}}$. Through appropriate choice of the order $n$ and modulation frequency $\omega_{\mathrm{m}}$, it is possible to amplify the resulting signal by an eigenmode of the cantilever. This approach has been termed harmonic mixing and was exploited recently to tune the imaging resolution in Kelvin probe force microscopy [21. In case of the fundamental harmonic, $n=1$, harmonic mixing is equivalent to heterodyne [20, 53] and sideband (de)modulation [54, 55] techniques.

The transfer functions derived from the baseband response of the cantilever are indispensable in the design of feedback loops. Empirical tuning by trial and error should be avoided, even more so when feedback loops depend on each other. In case of PLL-based excitation, it is crucial to include the influence of the cantilever for low quality factors.

The separation of cantilever response into a steadystate and transient solution calls for an obvious application in the optimization of AFM simulators. Such simulators [49, 50, 56, 59] are used to understand the influence of feedback loops and the operator on the measurement, 
can explain imaging artifacts 60, 61] and correlate experiment and theory 62. Moreover, they have a potential use in the training and education of new AFM users [58, 63.

There is a tradeoff between accuracy of the simulation and the computational power required. The prevalent approach [49, 50, 58, 59] is the direct numerical solution of the equation of motion, eq. (2). While being the most general and accurate, however, the direct solution is computationally very demanding, because for numerical stability the time step must be chosen significantly below the oscillation period. On the other hand, the static solution alone, given by eqs. 22a to 22d, already allows one to calculate AFM images under ideal conditions, i.e., neglecting the effects of feedback loops and possible modifications of the AFM tip and surface. The dynamics can be considered on top of the static solution by a numerical solution of the baseband equation of motion, eq. (52), which accurately models the cantilever dynamics relevant for AM- and FM-AFM. Since high-frequency dynamics are neglected in this equation, the time steps for the integration can be chosen much larger compared to direct integration of eq. (2). Additionally, for numerical investigations, it is often possible to calculate $\left\langle k_{\mathrm{ts}}\right\rangle$ and $\left\langle\gamma_{\mathrm{ts}}\right\rangle$ in advance. For this reason, an AFM simulator built around eq. (52) can be implemented very efficiently.

\section{SUMMARY}

This work provides compact solutions of the steadystate and transient behavior of an AFM tip under the influence of external forces. Whereas the steady-state behavior has been studied extensively before for AM-AFM and FM-AFM individually, a unifying derivation of the underlying solution based on the harmonic approximation was only given recently 15 .

We have derived the transient behavior of the deflection signal from a perturbation of this steady-state solution. In essence, it was shown that transients are linked to the time evolution of Fourier coefficients of the tipsample force and their modulation by the cantilever response function.

We have also described the complex baseband behavior and provided expressions of the resulting amplitude, phase, and frequency modulation. These were used to derive the transfer functions of the PLL and amplitude controller used for FM-AFM.

Our results show that a holistic treatment of the cantilever movement enables a deeper understanding of its behavior and reveals the many interconnections and similarities of dynamic AFM techniques used today and in future.

\section{ACKNOWLEDGMENTS}

The author greatly appreciates the fruitful and constructive discussions with Prof. Andreas Stemmer throughout the preparation of this manuscript.
[1] G. Binnig, C. Quate, and C. Gerber, Phys. Rev. Lett. 56, 930 (1986).

[2] R. García and R. Pérez, Surf. Sci. Rep. 47, 197 (2002).

[3] Y. Martin, C. C. Williams, and H. K. Wickramasinghe, J. Appl. Phys. 61, 4723 (1987).

[4] T. R. Albrecht, P. Grütter, D. Horne, and D. Rugar, J. Appl. Phys. 69, 668 (1991).

[5] J. P. Cleveland, B. Anczykowski, A. E. Schmid, and V. B. Elings, Appl. Phys. Lett. 72, 2613 (1998).

[6] B. Anczykowski, B. Gotsmann, H. Fuchs, J. P. Cleveland, and V. B. Elings, Appl. Surf. Sci. 140, 376 (1999).

[7] Y. Martin and H. K. Wickramasinghe, Appl. Phys. Lett. 50, 1455 (1987).

[8] Y. Martin, D. W. Abraham, and H. K. Wickramasinghe, Appl. Phys. Lett. 52, 1103 (1988).

[9] D. Nowak, W. Morrison, H. K. Wickramasinghe, J. Jahng, E. Potma, L. Wan, R. Ruiz, T. R. Albrecht, K. Schmidt, J. Frommer, D. P. Sanders, and S. Park, Science Advances 2, e1501571 (2016).

[10] M. Nonnenmacher, M. P. O'Boyle, and H. K. Wickramasinghe, Appl. Phys. Lett. 58, 2921 (1991).

[11] S. Kitamura and M. Iwatsuki, Appl. Phys. Lett. 72, 3154 (1998).

[12] A. San Paulo and R. Garcia, Phys. Rev. B 66 (2002).

[13] F. J. Giessibl, Phys. Rev. B 56, 90128 (1997).
[14] J. E. Sader, T. Uchihashi, M. J. Higgins, A. Farrell, Y. Nakayama, and S. P. Jarvis, Nanotechnology 16, S94 (2005).

[15] H. Söngen, R. Bechstein, and A. Kühnle, J. Phys.: Condens. Matter 29, 274001 (2017).

[16] L. Nony, R. Boisgard, and J.-P. Aimé, J. Chem. Phys. 111, 1615 (1999).

[17] R. Garcia and A. San Paulo, Phys. Rev. B 60, 4961 (1999).

[18] R. Garcia and A. San Paulo, Phys. Rev. B 61, R13381 (2000).

[19] R. Garcia and E. T. Herruzo, Nat. Nanotechnol. 7, 217 (2012).

[20] Y. Sugawara, L. Kou, Z. Ma, T. Kamijo, Y. Naitoh, and Y. Jun Li, Appl. Phys. Lett. 100, 3104 (2012).

[21] J. L. Garrett, M. S. Leite, and J. N. Munday, ACS Appl. Mater. Interfaces 10, 28850 (2018).

[22] D. Platz, D. Forchheimer, E. A. Tholen, and D. B. Haviland, Nanotechnology 23, 265705 (2012).

[23] J. L. Garbini, K. J. Bruland, W. M. Dougherty, and J. A. Sidles, J. Appl. Phys. 80, 1951 (1996).

[24] J. Preiner, J. Tang, V. Pastushenko, and P. Hinterdorfer, Phys. Rev. Lett. 99, 046102 (2007).

[25] K. Kuchuk and U. Sivan, Beilstein J. Nanotechnol. 6, 149 (2015). 
[26] A. Rosa-Zeiser, E. Weilandt, S. Hild, and O. Marti, Meas. Sci. Technol. 8, 1333 (1997).

[27] C. A. Amo and R. Garcia, ACS Nano 10, 7117 (2016).

[28] D. Platz, E. A. Tholen, D. Pesen, and D. B. Haviland, Appl. Phys. Lett. 92, 3106 (2008).

[29] H. Hölscher, B. Gotsmann, W. Allers, U. D. Schwarz, H. Fuchs, and R. Wiesendanger, Phys. Rev. B 64, 075402 (2001).

[30] F. J. Giessibl, Appl. Phys. Lett. 78, 123 (2001).

[31] D. Ebeling and H. Hölscher, J. Appl. Phys. 102, 114310 (2007).

[32] H. Hölscher and U. D. Schwarz, Internat. J. Nonlin. Mech. 42, 608 (2007).

[33] J. E. Sader and S. P. Jarvis, Appl. Phys. Lett. 84, 1801 (2004).

[34] Y. A. Mitropolskii and N. Van Dao, "Applied Asymptotic Methods in Nonlinear Oscillations," Springer Netherlands, Dordrecht (1997).

[35] N. M. Krylov and N. N. Bogolioubov, "Introduction to non-linear mechanics: A free translation by Solomon Lefschetz of excerpts from two Russian monographs," Princeton University Press, Princeton, New Jersey (1949).

[36] L. Verlet, Phys. Rev. 159, 98 (1967).

[37] U. Dürig, O. Züger, and A. Stalder, J. Appl. Phys. 72, 1778 (1992).

[38] U. Dürig, H. R. Steinauer, and N. Blanc, J. Appl. Phys. 82, 3641 (1997).

[39] C. Loppacher, M. Bammerlin, F. Battiston, M. Guggisberg, D. Müller, H. R. Hidber, R. Lüthi, E. Meyer, and H. J. Güntherodt, Appl. Phys. A 66, S215 (1998).

[40] Y. Mitani, M. Kubo, K.-i. Muramoto, and T. Fukuma, Rev. Sci. Instrum. 80, 083705 (2009).

[41] B. Schlecker, M. Dukic, B. Erickson, M. Ortmanns, G. Fantner, and J. Anders, IEEE Trans on BioCAS 8, 206 (2014).

[42] K. Miyata and T. Fukuma, Beilstein J. Nanotechnol. 9, 1844 (2018).

[43] R. E. Best, Phase-locked loops, 5th ed. (McGraw-Hill, 2003).

[44] B. I. Kim, Rev. Sci. Instrum. 75, 5035 (2004).
[45] G. B. Kaggwa, J. I. Kilpatrick, J. E. Sader, and S. P. Jarvis, Appl. Phys. Lett. 93, 011909 (2008).

[46] T. Fukuma, S. Yoshioka, and H. Asakawa, Rev. Sci. Instrum. 82, 3707 (2011).

[47] F. M. Gardner, Phaselock techniques, 3rd ed. (WileyInterscience, 2005).

[48] W. F. Egan, Phase-lock basics, 2nd ed. (WileyInterscience, 2007).

[49] J. Polesel-Maris and S. Gauthier, J. Appl. Phys. 97, 044902 (2005).

[50] L. Nony, A. Baratoff, D. Schär, O. Pfeiffer, A. Wetzel, and E. Meyer, Phys. Rev. B 74, 235439 (2006).

[51] J. Lübbe, M. Temmen, P. Rahe, and M. Reichling, Beilstein J. Nanotechnol. 7, 1885 (2016).

[52] Y. Miyahara and P. Grutter, Appl. Phys. Lett. 110 (2017).

[53] J. L. Garrett and J. N. Munday, Nanotechnology 27, 245705 (2016).

[54] U. Zerweck, C. Loppacher, T. Otto, S. Grafström, and L. M. Eng, Phys. Rev. B 71, 125424 (2005).

[55] T. Wagner, H. Beyer, P. Reissner, P. Mensch, H. Riel, B. Gotsmann, and A. Stemmer, Beilstein J. Nanotechnol. 6, 2193 (2015).

[56] G. Couturier, J.-P. Aimé, J. Salardenne, and R. Boisgard, Eur. Phys. J. Appl. Phys. 15, 141 (2001).

[57] T. Trevethan, M. Watkins, L. N. Kantorovich, and A. L. Shluger, Phys. Rev. Lett. 98, 028101 (2007).

[58] D. Kiracofe, J. Melcher, and A. Raman, Rev. Sci. Instrum. 83, 013702 (2012).

[59] J. Tracey, F. Federici Canova, O. Keisanen, D. Z. Gao, P. Spijker, B. Reischl, and A. S. Foster, Computer Physics Communications 196, 429 (2015).

[60] L. Nony, F. Bocquet, C. Loppacher, and T. Glatzel, Nanotechnology 20, 264014 (2009).

[61] L. Nony, A. S. Foster, F. Bocquet, and C. Loppacher, Phys. Rev. Lett. 103, 36802 (2009).

[62] F. Castanié, L. Nony, S. Gauthier, and X. Bouju, J. Phys. Chem. C 117, 10492 (2013).

[63] H. V. Guzman, P. D. Garcia, and R. Garcia, Beilstein J. Nanotechnol. 6, 369 (2015). 